\title{
Numerical Analysis of Self-wastage Phenomena Caused by Sodium-Water Reaction in Sodium-Cooled Fast Reactor through Simulant Experiment
}

\author{
Sunghyon Jang ${ }^{1}$, Takashi Takata ${ }^{1}$ and Akira Yamguchi ${ }^{2}$ \\ 1. Graduate School of Engineering, Osaka University, Osaka 565-0871, Japan \\ 2. Graduate School of Engineering, University of Tokyo, Ibaraki 319-1188, Japan
}

Received: February 12, 2015 / Accepted: May 04, 2015 / Published: June 30, 2015.

\begin{abstract}
A water leakage on the surface of heat transfer tube in a steam generator of sodium-cooled fast reactor causes SWR (sodium-water reaction). The SWR damages the leak surface and gives rise to the leak enlargement. Most of initial leakage starts from micro leak (less than $0.5 \mathrm{~g} / \mathrm{s}$ ). However, the leak rate increases more than two orders of magnitude and the resultant leak damages surrounding heat transfer tubes and it brings secondary failure of the heat transfer tube. Evaluation of the leak enlargement is necessary to assess the leak rate increase, so that evaluate the possibility of secondary failure. In this study, a simulant experiment, which uses neutralization reaction, is proposed to reproduce the leak enlargement. To examine the feasibility of the experiment, numerical simulations are carried out. From the result, a funnel-shaped nozzle enlargement is observed and the shape similar to the shape of the enlarged nozzle from the SWAT (sodium-water reaction test loop) experiment.
\end{abstract}

Key words: Sodium-cooled fast reactor, self-wastage phenomena, sodium-water reaction, simulant experiment, CFD (computational fluid dyanamics).

\section{Introduction}

SWR (sodium-water reaction) is a design basis accident of a SFR (sodium-cooled fast reactor). A breach of the heat transfer tube in a steam generator results in contact with liquid sodium with water vapor. Typical phenomena are deterioration of the tube wall material and its removal due to the corrosion and the erosion. As results, the breach is enlarged at its initial site, and it brings a sharp increase of leak rate. The enlargement of leak caused by the SWR is called self-wastage phenomena. According to experimental researches, it has been observed that, even though the initial leak rate is small (less than $0.05 \mathrm{~g} / \mathrm{s}$ ), the leak rate increases more than two orders of magnitude over a period of about $10 \mathrm{~s}[1,2]$. Thus, there is a danger that

Corresponding author: Sunghyon Jang, Ph.D. candidate, research fields: thermal hydraulics and CFD of sodium fast reactor. E-mail: jang_s@qe.see.eng.osaka-u.ac.jp. the resultant leak rate caused by the self-wastage might be in the region of a small leak, or even an intermediate leak which would then give rise to problems of target wastage. Therefore, it is an important issue to understand the behavior of micro-leak for the design of the leak detector and shutdown systems. So far, the phenomena of self-wastage have been studied experimentally by several investigators [3-7]. These experimental studies focused on deriving the relationship between the initial leak rate and the resultant leak rate. However, these mock-up tests had uncertainties and limitations since the SWR is complex, and the experimental measurement technology is not well matured enough to unroll the reaction mechanism in detail.

Recently, numerical approach using multi-dimensional analysis code such as SERAPHIM (sodium-water reaction analysis: physics of interdisciplinary multi-phase flow) code was 
attempted to depict the self-wastage phenomena [8]. However, these numerical approaches have some limitations to simulate the phenomena due to complexity of the self-wastage phenomena and large computational load for the analysis.

So we suggested a new method to assess the self-wastage phenomena through a simulant experiment. This new approach focuses on reproducing the self-wastage phenomena. Since the self-wastage phenomena are attributed to a chemical reaction takes place near the exit of the leak. We assumed that, it is able to evaluate the damage on the surface of the leak by making the neutralization reaction with $\mathrm{HCl}$ (hydrochloric acid) solution and $\mathrm{NaOH}$ (sodium hydroxide) solution.

In this paper, at first, we design the concept of the simulant experiment. Then, as a preliminary investigation, numerical analysis is carried out to validate the feasibility of the method and to decide experimental condition. In the analysis, governing equations of concentrations of acid and alkali are implemented into a commercial CFD (computational fluid dynamics) tool, Fluent Ver.6.3.26.

\section{Sketch of the Simulant Experiment}

\subsection{SWAT (Sodium-Water Reaction Test Loop) Experiment}

Experimental studies on the self-wastage phenomena had been carried out to simulate SFR prototype MONJU steam generators tubes in the small leak SWAT by PNC (Power Reactor and Nuclear Fuel Development Cooperation), Japan [9]. Slit-type nozzle has the same thickness as the actual heat transfer tube were used to the micro-leak behavior in the Monju steam generator. Through the experiment, the relationship between the average leak rate, the sodium temperature and the self-wastage rate were derived. Also it was reported that, the enlarged orifice diameter of the leaks were in a certain range, with no relations to the average leak rates, the materials, the temperatures, and the initial nozzle shape [10]. Generally, however, it is expected that, the self-wastage phenomena are complex that many parameters such as leak rate, sodium temperature, leak geometry and so on, are related. In addition, high chemical activity of the reaction and opacity of sodium in the liquid state make hard to evaluate quantitative analysis of the self-wastage phenomena.

\subsection{Simulant Material}

To elucidate the self-wastage phenomena and evaluate quantitative analysis, simulant experiment is designed. The self-wastage phenomena are attributed to the interaction between the wall material and the corrosion and the erosion by the SWR. This combination of the erosive and corrosive effects of the SWR is so complicated that it is hard to evaluate the self-wastage phenomena quantitatively. However, from a phenomenological viewpoint, it can be said that, the self-wastage phenomena are attributed to an exothermic reaction which take places around the exit of the leak. Thus, the idea of adopting an exothermic reaction is proposed to simulate the self-wastage phenomena. The neutralization is an exothermic reaction when $\mathrm{HCl}$ and $\mathrm{NaOH}$ aqueous solution are mixed the following exothermic reaction takes place.

$$
\mathrm{HCl}_{(\mathrm{aq})}+\mathrm{NaOH}_{(\mathrm{aq})} \rightarrow \mathrm{NaCl}_{(\mathrm{aq})}+\mathrm{H}_{2} \mathrm{O}+\Delta H
$$

As a result of the reaction, the reaction heat $(\Delta H=$ $55.6 \mathrm{~kJ} / \mathrm{mol}$ ) releases. Paraffin wax $115 \mathrm{~F}$, which has a relatively low melting point $(115 \mathrm{~F}, 319.2 \mathrm{~K})$, is adopted as simulant to represent the tube wall. When the neutralization reaction takes places on the leak exit, it is expected that, the temperature in the reaction zone increases beyond the melting point of the wax so that the wax in that area is melted by the reaction heat. As the reaction continues, the enlargement advances through the nozzle wall. This procedure reproduces the crack enlargement by the self-wastage phenomena.

\subsection{Experimental Procedure}

Brief depiction of the self-wastage phenomena and the simulant experiment are described in Fig. 1. 


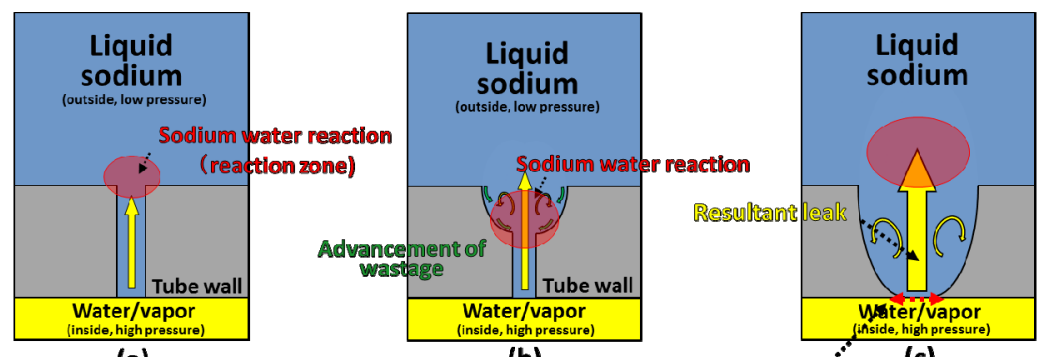

(a)

(b)
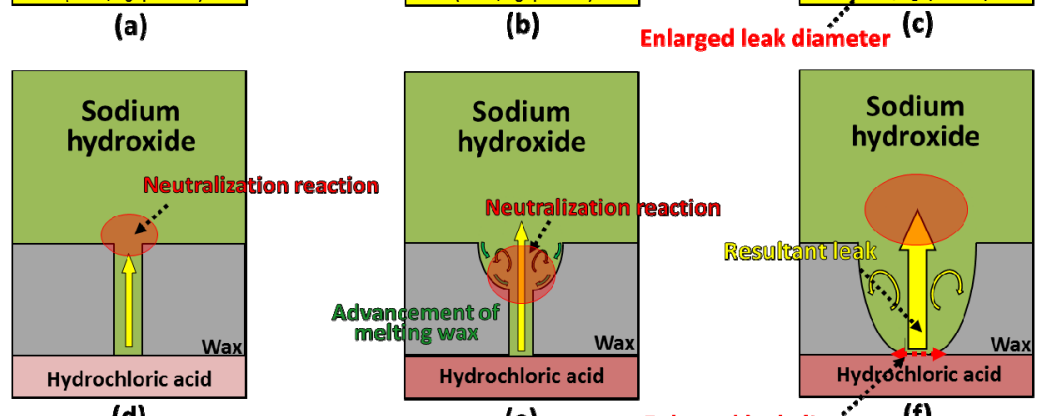

(d)

(e)

Enlarged leak diarieter (f)

Fig. 1 Overview of self-wastage and simulant experiment.

(1) The self-wastage phenomena are attributed to a chemical reaction, which is followed by reaction heat, takes place at the initial leak site;

(2) The wastage phenomena start from the sodium side and advance through the tube thickness. As the leak gets enlarged by the self-wastage, the reaction zone advances toward the water/vapor side. As a result of the enlargement, the corrosive reactants reach the tube wall. Thus, the sodium water reaction will be continued. The leak rate has stability in this stage;

(3) When the wastage develop to a certain depth to the water side, the remaining tube wall will be removed by high-inner pressure of the vapor, so that the leak rate will be sharply increased. Then the sodium water reaction zone migrates to the sodium side, so that the self-wastage is mitigated;

(4) $\mathrm{HCl}$ and $\mathrm{NaOH}$ are used as simulant. Paraffin wax $115 \mathrm{~F}$ is chosen as nozzle simulant to represents the heat transfer tube. In the experiment, $\mathrm{HCl}$ solution is injected into the reaction tank which is filled with $\mathrm{NaOH}$ solution through the paraffin wax nozzle, so that the neutralization reaction would occur at the exit of the nozzle and the reaction heat will be released;

(5) If the temperature of the surface of the wax exceeds the melting point of the paraffin wax, the surface will be melted, and the nozzle will be enlarged. As the wax is melted, new reaction zone advanced through the wall;

(6) When the enlargement reaches to the $\mathrm{HCl}$ side, investigate the geometry of the enlarged nozzle, the effect of the self-wastage on the heat transfer tube could be evaluated.

Fig. 2 shows a schematic experimental apparatus for the simulant experiment. Experimental setup consists of a reaction tank, a paraffin wax nozzle and a solution supply system. In this study, as a preliminary analysis, numerical analysis are carried out to evaluate the feasibility of the simulant experiment and to decide the experimental conditions by using commercial CFD software ANSYS Fluent 6.3.36.
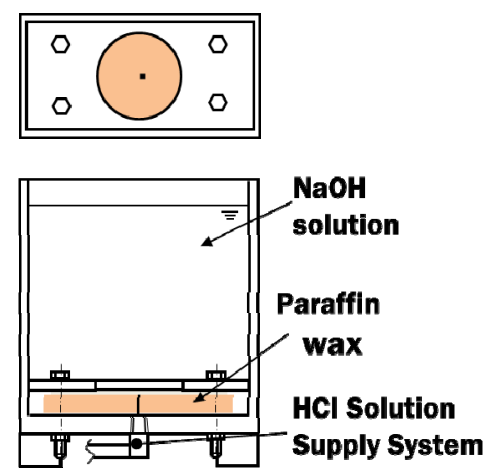

Fig. 2 Scheme of experimental apparatus. 


\section{Numerical Feasibility Analysis}

\subsection{Governing Equations}

A two-dimensional unsteady flow of mass transfer with a chemical reaction has been calculated in this study. The conservation equation for chemical species is given by Eq. (2):

$$
\frac{\partial}{\partial t}\left(\rho Y_{i}\right)+\nabla \cdot\left(\rho \bar{v} Y_{i}\right)=-\nabla \cdot \vec{J}_{i}+R_{i}
$$

where, $R_{i}$ is the net rate of production of mass of species; $Y_{i}$ is the per unit volume by chemical reaction; $\vec{J}_{i}$ is the diffusion flux of species $i$, which arises due to concentration gradients. For the net reaction rate, the laminar finite-rate model is adopted. Chemical species are computed based on the Arrhenius expression [11]. The net source of chemical species $i$ due to the reaction is computed as the sum of the Arrhenius reaction sources over the $N_{R}$ reaction which the species participate in Eq. (3):

$$
R_{i}=M_{w, i} \sum_{r=1}^{N_{R}} R_{i, r}
$$

where, $M_{w, i}$ is the molecular weight of species $i$ and $R_{i, r}$ is the Arrhenius molar rate of creation/destruction of species $i$ in reaction $r$. Reaction occur in the continuous phase at wall surfaces. The molar rate of species $i$ in reaction is given by Eq. (4):

$$
R_{i, r}=k_{F}\left[C_{j, r}\right]^{\left(\eta_{j, r}^{\prime}+\eta_{j, r}^{\prime \prime}\right)}
$$

where, $k_{F}=$ rate constant for reaction; $C_{j, r}=$ molar concentration of species $j$ in reaction $r ; \eta_{j, r}^{\prime}=$ rate exponent for reactant species $j$ in reaction $r ; \eta_{j, r}^{\prime \prime}=$ rate exponent for product species $j$ in reaction $r$.

The rate constant can be computed from the Arrhenius equation shown as below:

$$
k_{r}=A_{r} e^{-E_{a} / R T}
$$

where, $A_{r}=$ pre-exponential factor $\left(\mathrm{mol}^{-1} \cdot \mathrm{m}^{3} \cdot \mathrm{s}^{-1}\right) ; E_{a}=$ activation energy for the reaction $\left(\mathrm{J} \cdot \mathrm{mol}^{-1}\right) ; R=$ universal gas constant $\left(\mathrm{J} \cdot \mathrm{mol}^{-1} \cdot \mathrm{K}^{-1}\right)$.

\subsection{Numerical Approach}

Numerical approach to evaluate the self-enlargement of the leak consists of the three parts: construction of analytical model; wastage rate evaluation using local thermal hydraulic properties; remeshing process is based on the local wastage rate.

It requires large computational load to carry out a calculation during the whole time span of the phenomena. According to experimental results, after wastage starts from the sodium side, the leak rate stay almost unchanged until the thin edge of the wall is removed. Thus, the wastage phenomenon during this period can be regarded as steady. Therefore, instead of transient numerical calculations which consider the whole series of event at one time, a stepwise calculation is designed. At first, a transient calculation is carried out over a small period time until it reaches the steady condition. Then, the amount of the wastage rate is evaluated from the thermal properties from the numerical calculation, and then a new analytical mesh model is reconstructed. Fig. 3 shows the numerical procedure of the simulant experiment.

Step I: Determining an initial nozzle size: in order to achieve dynamic similarity between the SWAT experiment and the numerical analysis, Re number of the analysis is decided to be equal to that of the SWAT-2 experiment. The nozzle geometry is decided to have the same as aspect ratio of the real nozzle;

Step II: Investigation local thermal properties and evaluate the amount of the melting wax: carry out an unsteady numerical simulation for a certain period until it reaches steady state. Then obtain thermal properties such as temperature contour in the wax and species distribution at the exit of the nozzle. In real phenomena, the crack progresses according to the crystal grain boundary of the material. It is assumed that, the wax also melted according to the contour line of the melting point of the wax;

Step III: Remeshing based on the melting wax: the new mesh grid is produced by changing solid cells to fluid cells according to the temperature contour of the melting point which is obtained from the Step II; 

Fast Reactor through Simulant Experiment
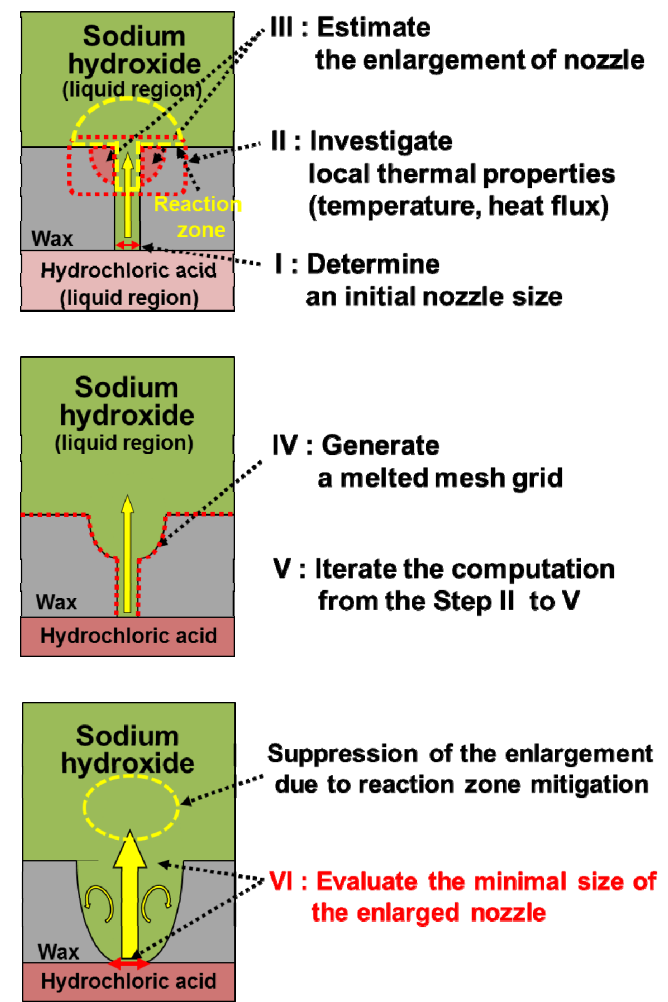

Fig. 3 Numerical procedure for simulant experiment.

Step IV: Iteration from the Step II to Step III: iterate from the Step II to Step III until the thickness of the remained wax on $\mathrm{HCl}$ side become $15 \%$ of the original thickness. This value is decided from the general viewpoint of probabilistic fracture mechanics of the failure of the heat transfer tube under high pressure;

Step V: Evaluating the geometry of the enlarged nozzle: when the thickness of the region of melted wax reaches the $85 \%$ of the initial thickness, investigate the geometry of the enlarged nozzle on the sodium side.

\subsection{Analytical Model and Conditions}

For the numerical calculation, a commercial software ANSYS Fluent 6.326 was used. Table 1 shows the experimental conditions of the SWAT-2 and the analytical conditions for simulant experiment [12]. As the same as the SWAT-2 experiment, a slit-type artificial crack is adopted as initial leak. Fig. 4 shows two-dimensional analytical region and its mesh arrangements. The region contains the reaction tank,
Table 1 Experiment and analytical conditions of SWAT-2.

(a) SWAT-2 experiment conditions

\begin{tabular}{ll}
\hline Experimental conditions & \\
\hline Tube material & $2.25 \mathrm{Cr}-1 \mathrm{Mo}$ \\
Tube thickness & $3.84 \mathrm{~mm}$ \\
Sodium temperature & $470{ }^{\circ} \mathrm{C}$ \\
Sodium pressure & $1.47 \times 10^{-1} \mathrm{MPa}$ \\
Steam temperature & $470{ }^{\circ} \mathrm{C}$ \\
Steam pressure & $12.8 \mathrm{MPa}$ \\
Initial crack width & $15 \mu \mathrm{m}$ \\
Initial leak rate & $3.4 \times 10^{-4} \mathrm{~g} / \mathrm{s}$ \\
Average leak rate & $2.5 \times 10^{-5} \mathrm{~g} / \mathrm{s}$ \\
\hline
\end{tabular}

(b) Analytical conditions for simulant

\begin{tabular}{ll}
\hline Initial condition & \\
\hline Temperature & $298 \mathrm{~K}$ \\
Fluid & $\mathrm{NaOH}$ \\
Concentration & $5.0 \mathrm{~mol} / \mathrm{L}$ \\
\hline Boundary condition & \\
\hline Inlet & $\mathrm{HCl}$ \\
Fluid & $0.05 \mathrm{~m} / \mathrm{s}$ \\
Velocity & $5.0 \mathrm{~mol} / \mathrm{L}$ \\
Concentration & \\
\hline Analytical condition & 9,961 \\
\hline Number of cells & $5.0 \mu \mathrm{s}$ \\
\hline
\end{tabular}

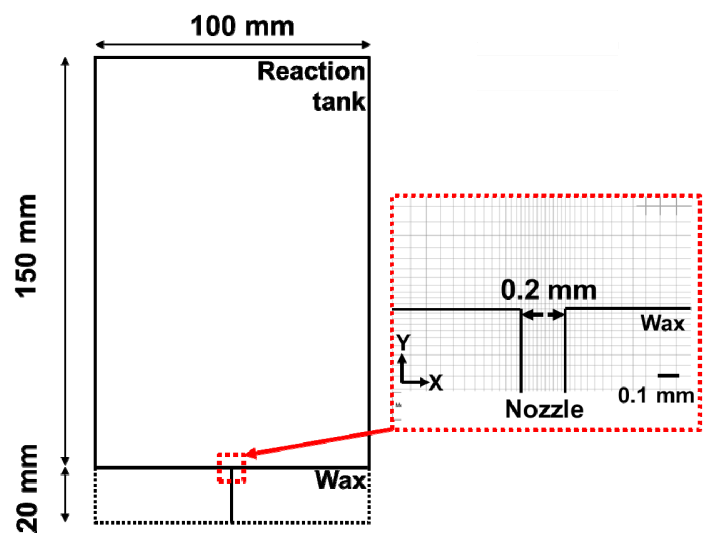

Fig. 4 Analytical region for initial calculation.

the paraffin wax and the nozzle. The thickness of the wax in the simulant material is decided as $2 \mathrm{~mm}$ from the viewpoint of the convenience in manufacturing. The width of the nozzle is determined to have the same aspect ratio with the crack of the SWAT-2 experiment so that the geometrical similarity is achieved between the real prototype and the simulant material. As shown in Fig. 4, the width of the initial nozzle is divided 
equally into 11 cells (I). The meshes of the other region are divided into 111 (I) $\times 59(\mathrm{~J})$ for reaction tank, 50 (I) $\times 42(\mathrm{~J})$ for the wax, and each element increases with the ratio of 1.1. The total number of the meshes is approximately 10,000 . In order to attain the dynamic similarity of the flow between the two models, the initial leak rate in the simulant experiment is decided to have the same Reynolds number with the real condition. For the inlet boundary, constant inlet velocity is adopted, and the inlet velocity is decided to have the same Reynolds number with the real condition had constant flow rate $\left(2.35 \times 10^{-5} \mathrm{~g} / \mathrm{s}\right)$ and the outlet boundary, constant pressure condition is used. The side walls of the reaction tank are the free-slip condition and the heat exchange between the fluids, and the surface of the wax on the top of the wax is considered.

\section{Results and Discussion}

\subsection{Analysis of the Initial Model}

Fig. 5 shows the numerical results of the initial model at several different seconds. The red line on the temperature contours indicates the melting point of the paraffin wax $(319.26 \mathrm{~K})$. The temperature inside the red line exceeds $319.26 \mathrm{~K}$. Accordingly, it is expected that, the wax in this area would be melted by the reaction heat. It is shown that, as time passes, the area, where temperature is higher than the melting point become smaller and located beside the nozzle exit.

In order to check that the calculation reaches steady state, transient temperature distribution on the top of the wax is obtained in Fig. 6. The temperature has slight changes less than $0.5 \mathrm{~s}$, however, temperature changes on the top of the wax do not distinguish from $0.5 \mathrm{~s}$ to $10.0 \mathrm{~s}$.

Fig. 7 shows the temperature distribution on the top of the wax at $10 \mathrm{~s}$. To estimate the width of the melted area, the temperature distribution toward $\mathrm{X}$-direction on the surface wax $(\mathrm{Y}=2.0 \mathrm{~mm})$ is obtained. In Fig. 7, the red dot-dashed line indicates the melting point of the paraffin wax and black dotted area indicates the width of the nozzle $(0.2 \mathrm{~mm})$. It is shown that, the

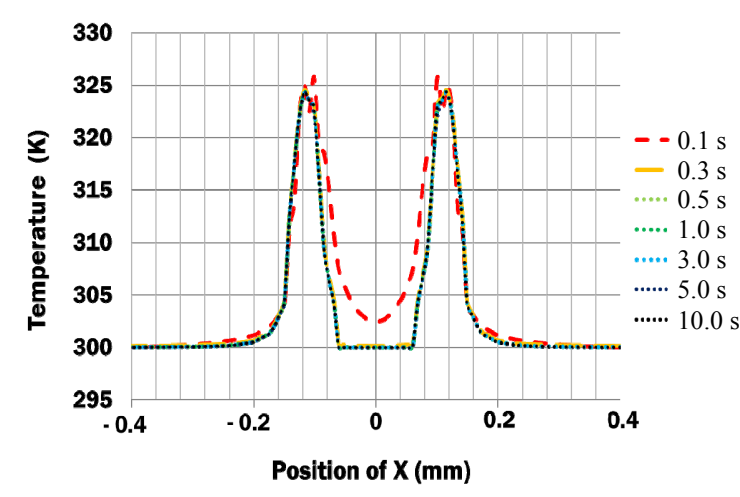

Fig. 5 Temperature around nozzle at different times.
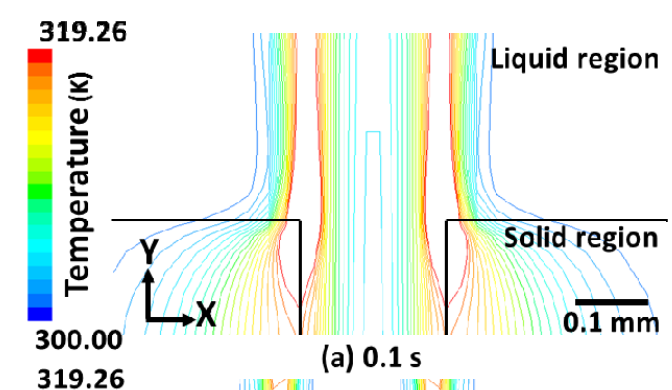

(a) $0.1 \mathrm{~s}$
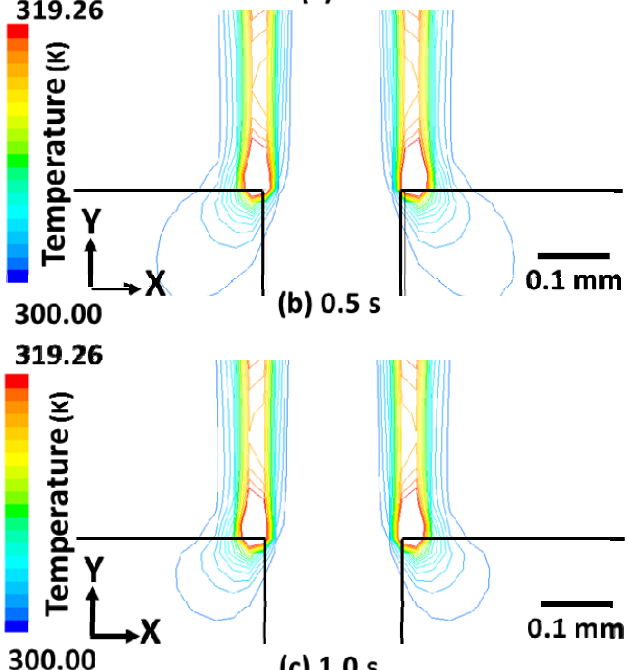
300.00

(c) $1.0 \mathrm{~s}$

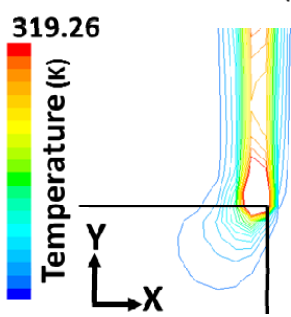

300.00

(d) $5.0 \mathrm{~s}$

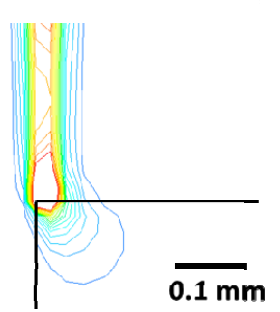

Fig. 6 Temperature on top of wax at different times: (a) estimated melting area; (b) new remeshed grid.

temperature of the wax surface right beside the nozzle increased to the melting point. The width of this area is approximately $0.035 \mathrm{~mm}$. Since the wax would be 


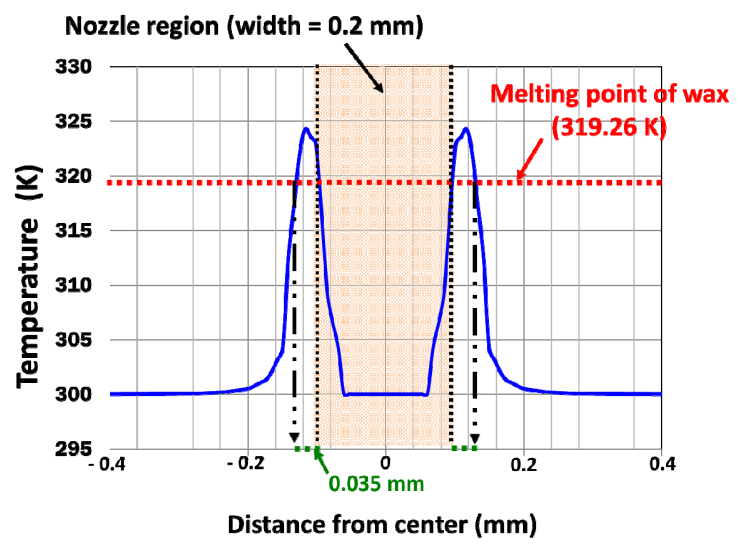

Fig. 7 Temperature on surface of wax at $10 \mathrm{~s}$.

melted along the temperature contour line of the melting point, it is assumed that, the thickness and shape of the melted area are regarded the same as that of the contour line.

So the shape of the surface of wax after being melted by the reaction heat would be represented as black-dotted line in Fig. 8a. A newly remeshed grid is obtained as shown in Fig. 8b by exchanging the solid (wax) cells to fluid cells in the melted area. These procedures mentioned as the Steps II and III are iterated until the remaining thickness of the wax become $15 \%$ of the initial thickness.

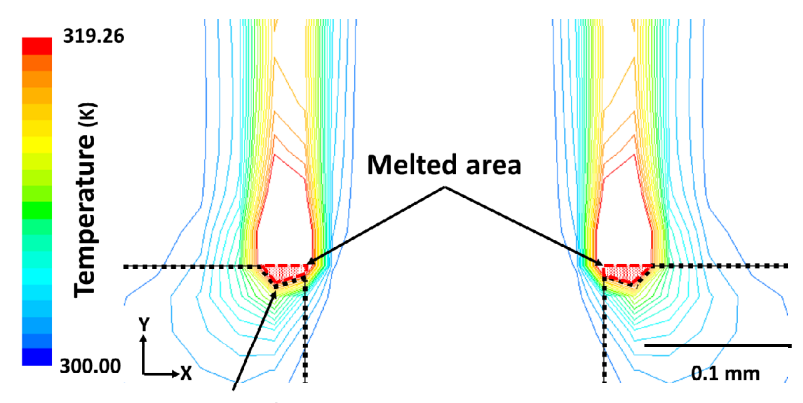

New grid surface

(a)

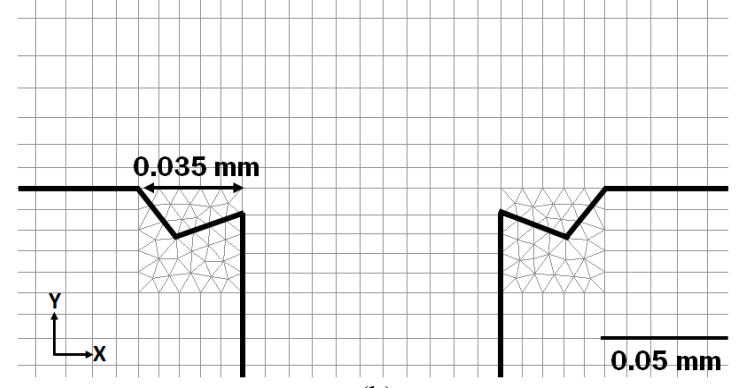

(b)

Fig. 8 Remeshing grid for new calculation.

\subsection{Further Numerical Analysis}

Using the newly remeshed grid in Fig. 8 further calculations are performed under the same numerical condition of the initial calculation. In total 75 calculations until the thickness of the remained wall on sodium side become $3 \mathrm{~mm}$. Fig. 9 shows the outline of the remeshed grid that are taken among the 75 calculations. As shown in the early states, the enlargement of the nozzle is noticeable in the both $\mathrm{X}$ and Y-direction as calculation goes further. The results of the further calculations show that the nozzle enlarges in an unsymmetrical way. In other words, one side of the wax at the top of the wax has more enlargement than the other side. However, as the calculation proceeds, it seems that the shape of the enlarged nozzle
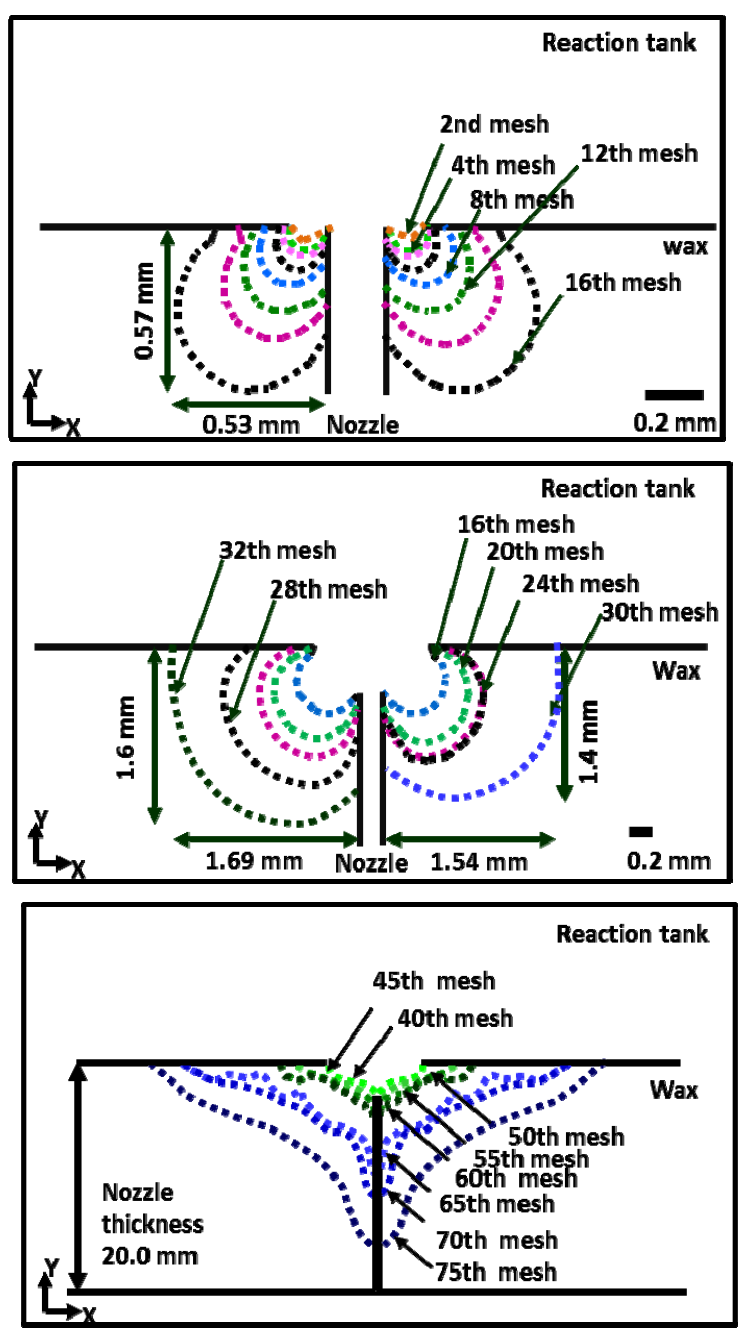

Fig. 9 Development of the enlargement of the nozzle. 
becomes almost symmetric again. Accordingly from the 40th calculation, even though the estimated enlarged nozzle shape is unsymmetrical, it is assumed that, the nozzle enlarged to have a symmetric shape. Through 75 calculations, it is found that, the enlargement advances until $15 \%$ of the initial tube thickness. So it is regarded that, the self-wastage phenomena are suppressed. Therefore, the numerical calculation is terminated.

\subsection{Evaluation of Geometry of the Enlarged Nozzle}

In the real condition, high pressure is working on the steam side, so that when the remaining tube wall reaches certain thickness, the remaining diaphragm of the wall would be removed by the steam jet. As a result, the width of the crack will be enlarged, and the remaining wall thickness will also be increased. In this study, it is assumed that, the when the remaining wall thickness become $15 \%$ of the original thickness, nozzle enlargement on $\mathrm{HCl}$ side take place, and the nozzle is regarded as penetrated. Fig. 10 shows the shape of the enlarged nozzle. The width of the complete enlarged nozzle is $3.52 \mathrm{~mm}$ which is 17.6 times as bigger than the initial width of the nozzle $(0.2 \mathrm{~mm})$. The shape of the enlarged nozzle is like a funnel shape with a larger opening toward the $\mathrm{NaOH}$ side.

Kuroha [13] reported that, the shapes of penetrated nozzle observed in the SWAT-2 test were like a funnel with a larger opening toward the sodium side. Fig. 11 shows the sectional morphology on complete self-wasted crack. In the present study, the aspect ratio of the nozzle and Re number are matched with the SWAT experimental data. As a result, a similar shape of the cross section of the enlarged nozzle is obtained.

Regarding the dimension of the enlarged nozzle, SWAT experimental data about the ratio of the diameter of the enlarged crack and the initial crack has ranged from 3.30 to 34.52 . In this study, the ratio of the enlarged nozzle width and the initial width was 17.6. It is hard to say that, the process of simulant experiment and that of a real experiment are exactly

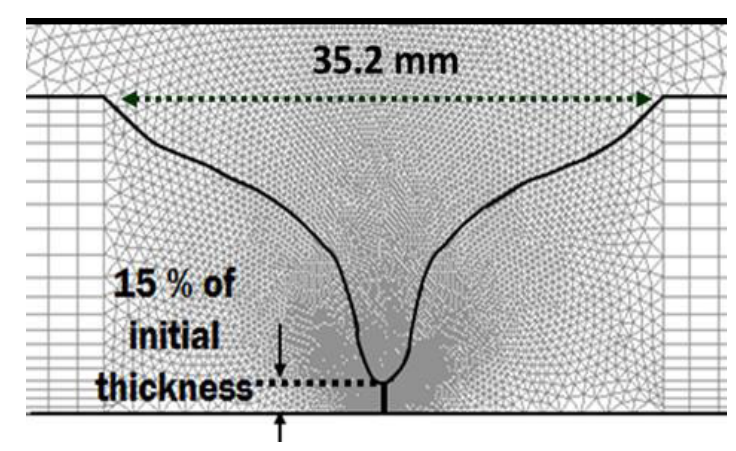

Fig. 10 Geometry of penetrated nozzle.

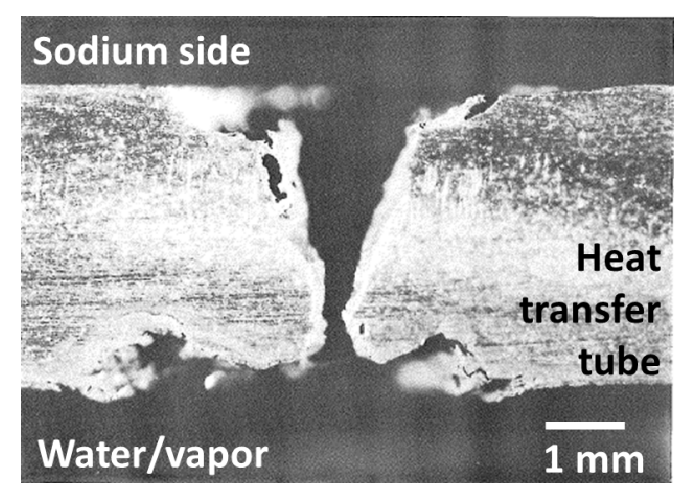

Fig. 11 Sectional morphology on self-wasted crack.

same, but from the ratio of the enlarged nozzle width and the initial width, it can be expected that, the simulant experiment shows similarity with the SWAT-2 experimental data.

\section{Conclusions}

The simulant experimental methodology of leak enlargement by the self-wastage phenomena is proposed. In the experiment, $\mathrm{HCl}, \mathrm{NaOH}$ aqueous solution and the paraffin wax are used as a simulant. The reaction heat released by the neutralization reaction with $\mathrm{HCl}$ and $\mathrm{NaOH}$ is used to reproduce the self-wastage phenomena. By injecting the $\mathrm{HCl}$ solution through a narrow wax nozzle, making the neutralization reaction near the outside of the nozzle. As a result of the release of the heat, the paraffin wax in the reaction zone would be melted, and the enlargement of the nozzle takes place.

To evaluate the feasibility of this methodology a computational analysis procedure is devised, and a preliminary numerical calculation is carried out. The 
enlargement of wax nozzle is estimated by using the temperature contour in the wax around the outside of the nozzle. Through the series of calculations, it is proved that, the propagation of the nozzle enlargement takes place due to the reaction heat of the neutralization and the enlargement advances toward $\mathrm{HCl}$ side. It is assumed that, when remained wall thickness becomes $15 \%$ of the initial thickness, the enlargement is terminated. The shape of the complete enlarged nozzle is a conical pit, and this shows a similarity with the cross section of the complete enlarged crack of SWAT-2 experiment. The ratio of the width of the enlarged nozzle and that of the initial nozzle is 17.6, and it also has a similar tendency with the real ratio that is from 3.30 to 34.52 .

The experimental condition is determined through this study. $\mathrm{HCl}$ solution is injected with $0.5 \mathrm{~m} / \mathrm{s}$ velocity through the wax nozzle (width $=0.2 \mathrm{~mm}$ ), and the flow has dynamic similarity with the flow in the real experiment (No. 2020) of SWAT-2 experiment. Also, it is proved that, there is a possibility to evaluate the effect of parameters such as injection rate, nozzle geometry in simulant experiment.

\section{References}

[1] Sandusky, D. W. 1975. Small-Leak Behavior Summary Report on Scoping Tests. Technical report.

[2] Hori, M. 1980. "Sodium/Water Reactions in Liquid Metal Fast Breeder Reactions." Atomic Energy Review 18 (3): 707-78.

[3] Green, D. A. 1976. "Small Leak Damage and Protection Systems in Steam Generators." In Proceedings of the International Conference of Liquid Metal Technology in Energy Production, 233-41.

[4] Quinet, J. L., and Lannou, L. 1979. "LMFBR Steam Generators: Behavior of Heat Exchange Tubes Faced with a Through Crack Resulting in Contact Between Sodium and Water." Nuclear Technology 46 (3): 582-90.

[5] Sandusky, D. W. 1974. "Behavior of Small Leaks in LMFBR Steam Generator." Trans. Am. Nucl. Soc. 19 (October): 106-7.

[6] Sandusky, D. W. 1976. "Small Leak Shutdown, Location and Behavior in LMFBR Steam Generators." In Proceedings of the International Conference on Liquid Metal Techonolgy in Energy Production, 226-32.

[7] Abe, Y., Shimoyama, K., and Kurihara, A. 2014. Development of Experimental Method for Self-wastage Behavior in Sodium-Water Reaction-Development of Test Rig (SWAT-2R) and Study for Experimental Procedure. JAEA (Japan Atomic Energy Agency)-Technology 2014-026.

[8] Takata, T., and Yamaguchi, A. 2002. Numerical Thermal-Hydraulic Study on Sodium-Water Phenomena-Development of Computational Code "SERAPHIM". Nuclear Cycle Development Institute Technical report.

[9] Kuroha, M., Inoue, S., Daigo, Y., and Sato, M. 1980. Preliminal Study of Micro-defect Self-wastage on 2 1/4Cr-1Mo Steel Nozzles for LMFBR Steam Generators-Studies of Micro-leak Sodium-Water Reactions (1). PNC ZN941 80-135.

[10] Kuroha, M., and Shimoyama, K. 1986. Micro-leak Behavior on LMFBR Monju Steam Generator Tube Material. PNC ZN941 86-027.

[11] Ansys. 2006. "Fluent 6.3 User's Guide.” Fluent Inc.

[12] Kuroha, M., Sasaki, K., Kawabe, H., Yamada, T., and Sato, M. 1982. "Study of Micro-defect Self-wastage Phenomena on LMBR Prototype Steam Generator's Tube-Studies of Micro-leak Sodium-Water Reactions (2)." In PNC TN941 82-101, Ibaraki: Japan Nuclear Cycle Development Institute, 23.

[13] Kuroha, M., Sasaki, K., Kawabe, H., Yamada, T., and Sato, M. 1982. "Study of Micro-defect Self-wastage Phenomena on LMBR Prototype Steam Generator's Tube-Studies of Micro-leak Sodium-Water Reactions (2)." In PNC TN941 82-101, Ibaraki: Japan Nuclear Cycle Development Institute, 57. 